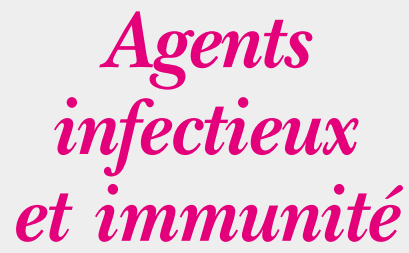

Durant l'été, deux articles, l'un paru dans Proc Natl Acad Sci USA et l'autre dans Cell, ont semblé apporter de l'eau au moulin de la thèse selon laquelle des rétrovirus pourraient être les facteurs déclenchants des maladies auto-immunes chez l'homme, la sclérose en plaques et le diabète de type 1 .

Perron et al. (Société BioMérieux de Lyon, Faculté de Médecine de Grenoble, University College de Londres) rapportent la caractérisation moléculaire partielle d'un virus décrit pour la première fois en 1989 à partir d'une culture de cellules leptoméningées obtenues du liquide céphalorachidien d'un malade atteint de sclérose en plaques. La séquence de l'ARN génomique de ce rétrovirus a été caractérisée par amplification PCR en utilisant des amorces dégénérées, l'ARN de virions extracellulaire étant utilisé comme matrice. Une séquence $p o l$ fut caractérisée et put être ainsi détectée dans l'ARN extracellulaire du plasma et du liquide céphalo-rachidien de malades atteints de sclérose en plaques mais non de témoins et de personnes atteintes d'autres maladies neurologiques [1]. Le nouveau virus a été désigné par les initiales MSRV (multiple sclerosis related virus); la séquence de sa transcriptase inverse le rapproche des oncovirus de type C. Un nombre déjà considérable de virus ont été, dans le passé, associés à la sclérose en plaques: notamment des paramyxoviridae et des herpesviridae, si bien qu'il faut certainement rester très prudent quant à la signification pathogénique de ce nouveau venu. Chez l'homme, on connaît au moins deux rétrovirus pathogènes capables, l'un et l'autre, d'entraîner des

\title{
Des rétrovirus à I'origine des maladies auto-immunes humaines ?
}

désordres neurologiques: les virus HTLV [2] et VIH.

Conrad et al., du laboratoire de Bernard Mach à Genève, ont, quant à eux, décrit une nouvelle séquence rétrovirale codant pour un superantigène qui pourrait jouer un rôle dans le déclenchement du diabète autoimmun (de type 1) [3]. En 1994, B. Conrad avait rapporté chez deux diabétiques l'enrichissement pancréatique en lymphocytes $\mathrm{T}$ ayant un gène $T C R$ avec un segment variable particulier V $\beta 7$ [4].

La stimulation de lymphocytes $\mathrm{T}$ par des préparations de membrane de pancréas diabétique, mais non de pancréas normal, stimulait la prolifération de cellules exprimant le même segment $\mathrm{V} \beta 7$, ce qui avait amené les auteurs à conclure à la présence d'un superantigène sur la membrane des cellules pancréatiques victimes de lésions d'insulite auto-immune. En effet, les superantigènes sont définis par leur propriété de stimuler l'activation polyclonale de lymphocytes $\mathrm{T}$ exprimant une chaîne V $\beta$ particulière lorsqu'ils sont présentés dans le contexte de molécules de classe II du complexe majeur d'histocompatibilité $(\mathrm{CMH})\left(\mathrm{m} / \mathrm{s} n^{\circ} 1\right.$, vol. 10, p. 87). Chez l'homme, on connaît bien de tels superantigènes d'origine bactérienne et virale, notamment rétrovirale, donc d'origine infectieuse et extérieurs à l'organisme. Chez la souris, un superantigène est codé par le génome du virus de la tumeur mammaire des souris (MMTV, murine mammary tumor virus). Conrad et al. [3] ont postulé l'existence d'un rétrovirus endogène humain qui commanderait la synthèse d'un superantigène, et ils ont recherché l'expression d'un tel virus, et surtout d'un tel superantigène, chez les diabétiques insulinodépendants (de type 1). Cette recherche a été accomplie en plusieurs étapes. Tout d'abord, une activité de transcriptase inverse a été détectée dans les leucocytes infiltrant les lésions d'insulite d'un pancréas diabétique, et non de tissu de personnes non diabétiques. Ensuite, des amorces oligonucléotidiques conservées ont permis d'amplifier par PCR un génome de type rétroviral de $8,6 \mathrm{~kb}$ au total, désigné par le sigle IDDMK $_{1,2} 22$. La région U3-R du LTR (long terminal repeat) 3' d'IDDMK ${ }_{1,2} 22$ fut amplifiée par PCR à partir du plasma de 10 diabétiques de type 1 récemment diagnostiqués, mais non de 10 sujets témoins de même âge et origine. En revanche, cette séquence est présente dans l'ADN génomique de sujets sains aussi bien que diabétiques, indiquant que IDDMK $_{1,2} 22$ est un rétrovirus endogène exprimé chez des diabétiques de type 1 et non chez des témoins.

D'un point de vue phylogénétique, $\mathrm{IDDMK}_{1,2} 22$ semble relié à MMTV et au rétrovirus endogène humain HERV-K10. Des expériences de transfection de monocytes dont l'expression des molécules de classe II était stimulée par l'interféron $\gamma$ avec divers vecteurs d'expression du génome rétroviral montrait enfin qu'une activité de type superantigène spécifique des chaînes V $\beta 7$ du TCR était codée par la partie N-terminale du gène d'enveloppe (env). Reste à comprendre le mécanisme d'activation de ce rétrovirus endogène et son rôle dans le déclenchement du diabète de type 1. On sait qu'existent plusieurs gènes de susceptibilité à cette maladie, notamment liés au gène de 
l'insuline $\left(\mathrm{m} / \mathrm{s} n^{\circ} 7\right.$, vol. 11, p. 1042) et au CMH (m/s $n^{\circ} 3$, vol. 7, p. 287). Certains de ces gènes pourraient-ils faciliter l'expression d'IDDMK ${ }_{1,2} 22$, peut-être à l'occasion d'une sensibilité particulière à une infection virale exogène déclenchante? Conrad et Mach proposent un modèle en deux temps où l'activation du rétrovirus endogène $\mathrm{IDDMK}_{1,2}$, dans les cellules

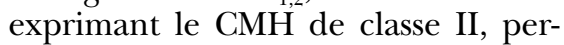
met l'expression du superantigène, qui induit alors une stimulation lymphocytaire $\mathrm{T}$ polyclonale systémique et massive.

Dans un deuxième temps, certains de ces lymphocytes activés, dotés d'un phénotype autoréactif, vont migrer vers les cellules $\beta$ du pancréas et enclencher un processus de destruction auto-immune sélective. La cause de l'activation supposée du superan- tigène est incertaine, et il pourrait en fait s'agir d'un phénomène secondaire à une agression auto-immune des cellules $\beta$ des îlots de Langerhans, d'une autre origine. Dans ce cas, la synthèse d'IDDMK ${ }_{1,2}$ pourrait néanmoins constituer un facteur d'aggravation de l'insulite. Enfin, on peut même imaginer que l'activation du rétrovirus endogène soit plus un marqueur qu'un acteur véritable de l'insulite [5].

Alors que le chemin à parcourir pour démontrer le rôle des rétrovirus récemment décrit reste considérable, il n'y a pas de doute que nombreuses sont les équipes à travers le monde qui recherchent aujourd'hui activement de telles séquences rétrovirales chez des malades atteints de maladies à composantes auto-immunes : polyar- thrite rhumatoïde, sclérose latérale amyotrophique, etc.

A.K.

1. Perron H, Garson JA, Bedin F, Beseme F, Paranhos-Baccala G, Komurian-Pradel F, Mallet F, Tuke PW, Voisset C, Blond JL, Lalandes B, Seigneurin JM, Mandrand B. Molecular identification of a novel retrovirus repeatedly isolated from patients with multiple sclerosis. Proc Natl Acad Sci USA 1997; 94: 7583-8.

2. Gessain A, de Thé G. Virus HTLV-I, leucémies $\mathrm{T}$ de l'adulte et neuromyélopathies chroniques. Med Sci 1987; 3 : 471-8.

3. Conrad B, Weissmahr RN, Böni J, Arcari R, Schüpbach J, Mach B. A human endogenous retroviral superantigen as candidate autoimmune gene in type I diabetes. Cell 1997; 90 : 30313.

4. Conrad B, Weidmann E, Trucco G, Rudert WA, Behboo R, Ricordi C, Rodriguez-Rilo H, Finegold $\mathrm{D}$, Trucco M. Evidence for superantigen involvement in insulin-dependent diabetes mellitus aetiology. Nature 1994; 371: 351-5.

5. Benoist C, Mathis D. Retrovirus as trigger, precipitator or marker? Nature 1997; 388: 833-4.

\section{BREVES}

Le rôle des récepteurs scavenger des macrophages dans l'athérosclérose et la susceptibilité aux infections. Les récepteurs scavenger de type A des macrophages ont des ligands très variés comme des bactéries, des polyanions et des LDL modifiées $\left(\mathrm{m} / \mathrm{s} n^{\circ} 7\right.$, vol. 11, p. 1043). Dans l'athérogenèse, l'accumulation de cholestérol dans les macrophages est la conséquence de l'internalisation des LDL modifiées par les récepteurs scavenger. Les souris dont les deux allèles du gène de l'apolipoprotéine $\mathrm{E}$ ont été invalidés présentent des lésions d'athérosclérose très importantes par rapport aux souris sauvages $\left(\mathrm{m} / \mathrm{s} n^{\circ} 10\right.$, vol. 8 , p. 1119). Chez ces souris, l'absence de récepteurs scavenger obtenue par invalidation génique se traduit par une réduction de la taille des lésions d'athérosclérose
[1]. In vitro, les macrophages qui n'expriment pas ces récepteurs scavenger internalisent moins bien les LDL modifiées par acétylation ou oxydation mais la clairance plasmatique de ces LDL modifiées est maintenue à un taux normal. Il existe donc une voie alternative à celle des récepteurs scavenger pour l'élimination des LDL modifiées plasmatiques. Cela suggère que c'est l'internalisation par les macrophages des LDL modifiées, et non leur clairance globale, qui intervient dans le développement de la plaque d'athérome. La persistence de lésions, même réduites, chez les souris sans apolipoprotéine $\mathrm{E}$ ni récepteur scavenger de type A indique que d'autres récepteurs $s c a$ venger de type MARCO, CD36, SRB$1 \mathrm{ou}$ macrosialine participent aussi à ces phénomènes. Les souris sans récepteur scavenger de type A présentent une susceptibilité accrue aux infections par Listeria monocytogenes et HSV-1. En ce qui concerne l'infection par Listeria, il semble que la susceptibilité des souris soit due à un défaut d'internalisation ou de destruction de la bactérie plutôt qu'à une différence dans la clairance plasmatique de la bactérie. Quel qu'en soit le mécanisme, les souris sans récepteur scavenger de type A ont démontré l'implication de ces récepteurs à la fois dans l'athérosclérose et dans la lutte contre les infections. Cela est sans doute à rapprocher des travaux de plus en plus nombreux faisant état d'un rôle direct du système immunitaire dans l'athérogenèse.

[1. Susuki H, et al. Nature 1997; 386: 292-5.] 